\title{
Terahertz time-domain spectroscopy of absorbing materials
}

\author{
F. Garet, ${ }^{* 1}$ B. Blampey, ${ }^{1}$ and J.-L. Coutaz ${ }^{1}$ \\ ${ }^{1}$ Laboratory IMEP-LAHC, UMR 5130 CNRS, University of Savoie 73376 Le Bourget du Lac Cedex, France
}

Received September 19, 2012; accepted September 26, 2012; published September 30, 2012

\begin{abstract}
In this paper, we study the noise-limited precision on the complex refractive index value of materials in the terahertz frequency range as determined by terahertz time-domain spectroscopy. As absorbing samples are almost opaque, transmission measurements are not suitable to determine the refractive index and reflection indices have to be employed instead, but at the expense of precision. We give rules to select the most adapted technique, i.e. in transmission or in reflection, for any given sample of arbitrary transparence.
\end{abstract}

TeraHertz (THz) time-domain spectroscopy (TDS) has been proposed at the end of the 80's [1] and is nowadays a well-established technique commonly used to characterize materials and devices in the very far infrared [2]. Commercial equipment is available [3], helping in spreading this technique throughout the world. When characterizing materials, one usually measures the transmission coefficients of flat homogeneous slabs. Different numerical methods [4-5] allow one to determine the complex refractive index $\tilde{n}=n+j \kappa$ of the sample material from the experimental data. If the material strongly absorbs the $\mathrm{THz}$ wave, the transmitted signal could be weaker than the sensitivity level of detection. In this later case, THz-TDS performed in reflection [6] is compulsory, at the expense of precision [7]. In this paper, we treat any case of samples, from totally opaque to transparent ones, in order to help researchers to choose a more precise measurement TDS technique, i.e. either in transmission or in reflection, versus the absorption and thickness of the sample.

For a plane wave incident field, the transmission and reflection coefficients $T$ and $R$ of the sample (homogeneous slab with flat and parallel surfaces) write:

$$
T=\frac{4 \tilde{n}}{(\tilde{n}+1)^{2}} \frac{\delta}{1-\left(\frac{\tilde{n}-1}{\tilde{n}+1}\right)^{2} \delta^{2}}, \quad R=\frac{\tilde{n}-1}{\tilde{n}+1} \frac{1-\delta^{2}}{1-\left(\frac{\tilde{n}-1}{\tilde{n}+1}\right)^{2} \delta^{2}},
$$

with $\delta=e^{j \frac{\omega}{c} \tilde{c} d} . \omega$ is the pulsation, $c$ the speed of light in vacuum, and $d$ the sample thickness. The actual value of $\tilde{n}$ is the solution of:

$$
T-T_{\text {meas }}=0 \text { or } R-R_{\text {meas }}=0 .
$$

\footnotetext{
*E-mail: garet@univ-savoie.fr
}

These equations have to be solved in the complex plane. Because of the oscillatory behavior of expressions (1), the solution of (2) is found by minimizing an error function [4]. The so-achieved value of $\tilde{n}$ is affected by errors whose origins are the sample itself (error on thickness, bad parallelism and inhomogeneity, bad positioning as compared to the reference mirror in reflection), the $\mathrm{THz}$ beam that is not parallel (or whose Rayleigh length is smaller than the sample thickness) and the experimental noise. In the most common case of photoconductive antennas, the total noise includes the laser noise, shot noise, Johnson noise and the noise in the electronics, and other noises, e.g. phase noise (fluctuation of air density in between the antennas). We suppose here that the sample is well known and that the TDS set-up is optimized. Thus we only treat the noise effect on the determination of $\tilde{n}$.

Let us first examine the noise of any complex experimental signal $\tilde{s}$. The measured value is equal to the actual value plus noise:

$$
s_{e x p} e^{j \phi_{e x p}}=s e^{j \phi}+s_{N} e^{j \phi_{N}},
$$

$s$ is the amplitude, $\phi$ is the phase, and the subscript $N$ indicates noise. As $s>s_{N}$, we easily get the standard deviations $\sigma_{s}=\Delta s$ and $\sigma_{\phi}=\Delta \phi$ :

$$
\Delta s=\sqrt{\overline{s_{N}^{2} \cos ^{2}\left(\phi_{N}-\phi\right)}}=\left(\overline{s_{N}^{2}} / 2\right)^{1 / 2}, \quad \Delta \phi=\frac{\Delta s}{s}
$$

where $\bar{X}$ is for average value of $X$.

We now apply these results to the reflection case. For the sake of simplicity, we consider moderately to strongly absorbing samples. Rebounds of the beam inside the sample are negligible and (1) simplify into:

$$
R=r e^{j \phi} \approx \frac{\tilde{n}-1}{\tilde{n}+1}
$$

The inversion of (5) is straightforward and leads to:

$$
n=\frac{1-r^{2}}{1-2 r \cos \phi+r^{2}}, \quad \kappa=\frac{2 r \sin \phi}{1-2 r \cos \phi+r^{2}}
$$

Through the derivative of (6) and the use of (4), we get: 


$$
\Delta n=\Delta \kappa=2 \frac{\left|\left(1+r^{2}\right) \cos \phi-2 r\right|+\left(1-r^{2}\right)|\sin \phi|}{\left(1-2 r \cos \phi+r^{2}\right)^{2}} \Delta r
$$

The last stage of the modeling concerns the calculation of $\Delta r$ from the experimental data. The experimental $R_{\text {exp }}$ coefficient is obtained by taking the ratio of the signal $S_{R}$ reflected by the sample (in THz-TDS, this signal is the Fourier transform of the reflected waveform) and of the reference signal $S_{\text {ref }}$ as reflected by a perfect mirror, both being affecting by noise:

$$
R_{\text {exp }}=\frac{S_{R}+S_{N R}}{S_{\text {ref }}+S_{\text {Nref }}} \approx R+\frac{S_{N R}-R S_{N r e f}}{S_{r e f}} .
$$

This relation between measured $R_{\text {exp }}$ and actual $R$ allows us to obtain $\Delta r$ :

$$
\Delta r=\sqrt{\left(R_{\text {exp }}-R\right)^{2}}=\frac{\sqrt{\left(\Delta s_{R}\right)^{2}+r^{2}\left(\Delta s_{r e f}\right)^{2}}}{s_{\text {ref }}} .
$$

To check the validity of the preceding equations, we proceed as follows: we measure the signal $S_{R}$ reflected by the sample, then we replace the sample by a perfect mirror, and we measure the reference signal $S_{r e f}$. This procedure is repeated several times. For the whole set of measured data, we calculate the average value of $R=r e^{j \phi}$ from where we get $\tilde{n}=n+j \kappa$ by using relations (6). From the calculated (using (9)) standard deviation $\Delta r$, we get the errors $\Delta n$ and $\Delta \kappa$ with (7).

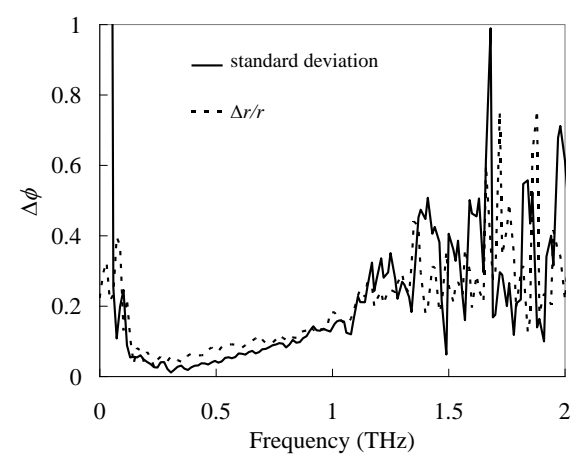

Fig. 1. Error on the phase, vs. frequency, in a reflection THz-TDS experiment: continuous line calculated from averaging over 12 measures, dashed line calculated with (4).

This procedure has been applied to a thick sample of high resistivity silicon. Fig. 1 shows the experimental error on the phase (standard deviation over 12 measurements) and the value obtained with relation (4). When the noise remains weak, i.e. in the frequency range $0.15 \sim 1.1 \mathrm{THz}$, the agreement is very good.

http://www.photonics.pl/PLP

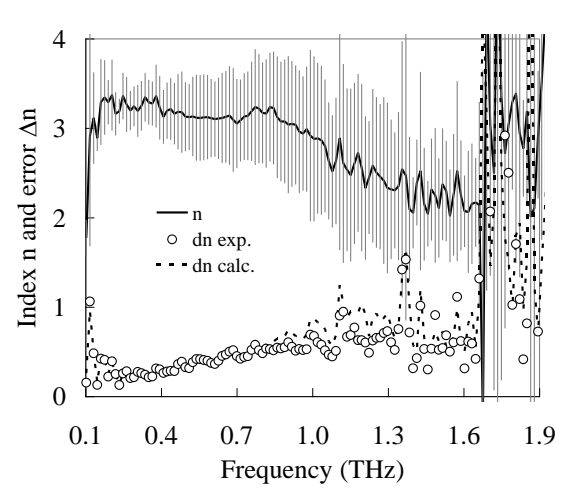

Fig. 2. Spectrum of the refractive index of high resistivity silicon measured by reflection THz TDS, together with the error $\Delta n$.

The refractive index $n$ of the same sample is plotted in Fig. 2 together with the error bars (in grey). The corresponding precision $\Delta n$ calculated with (7) is shown as a dashed curve, while open circles represent the standard deviation on $n$ directly calculated from the 12 sets of data. The agreement between these two values is good, validating relation (7). However, one can notice that the error is quite large, typically $20 \%$ at $1 \mathrm{THz}$. This is due to the weak reflected signal. In addition, $n$ deviates from the known value $(n=3.4)$ obtained from transmission TDS. This is certainly due to a small misalignment of the sample as compared to the reference mirror position.

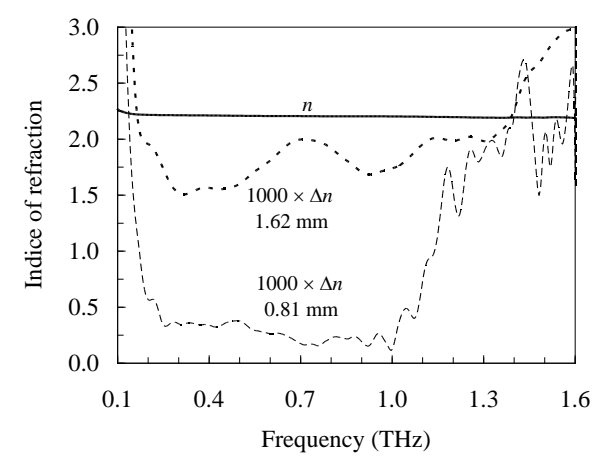

Fig. 3. Dispersion of the refractive index of Stycast glue in the THz range together with the noise-limited precision.

A similar study can be performed for transmission THzTDS. Useful relations are found in [4]. As an example, Fig. 3 shows the dispersion of the refractive index of a dry Stycast sample, which is a glue commonly used in cryogenic works. The index of refraction is $\sim 2.2$ in the $0.2 \sim 1.6 \mathrm{THz}$ studied range. The noise-limited precision on $n$ is excellent. For a $0.81-\mathrm{mm}$ thick sample, it is as good as $3 \times 10^{-3}$, i.e. $\sim 0.1 \%$, between $0.2 \sim 1 \mathrm{THz}$. At the extremities of the spectrum, the precision is worst due to the weaker incident $\mathrm{THz}$ power. For thicker samples (here $1.62 \mathrm{~mm}$ ), the precision is degraded because the transmitted signal is less intense. It is worth noticing that here the actual 
precision is not limited by the noise, but by the precision on the sample thickness or on the slab parallelism. An error $\Delta d$ on $d$ implies, at first order, the same relative error on $n$. For example, $\Delta d=10 \mu \mathrm{m}$ for a $d=1 \mathrm{~mm}$ thick sample leads to $\Delta n / n=1 \%$.

From the preceding results, it appears that the achieved precision depends both on the studied sample characteristics and on the measurement technique (reflection or transmission). Using a simplified model for the noise source in THz-TDS, which is described in [8] in the case of transmission, we are able to compute $\Delta r$ or $\Delta t$ versus $n$ and $\kappa$, and also versus $d$ in case of transmission. Thus, using relations (7), we calculate, versus the same parameters, $\Delta n$ and $\Delta \kappa$. As transmission depends on the sample thickness $d$ and on the frequency (relation (1)), we use $\beta=d \omega / c$ as parameter in the calculation. We neglect the Fabry-Perot effect as we are interested in the case of moderately to strongly absorbing materials.

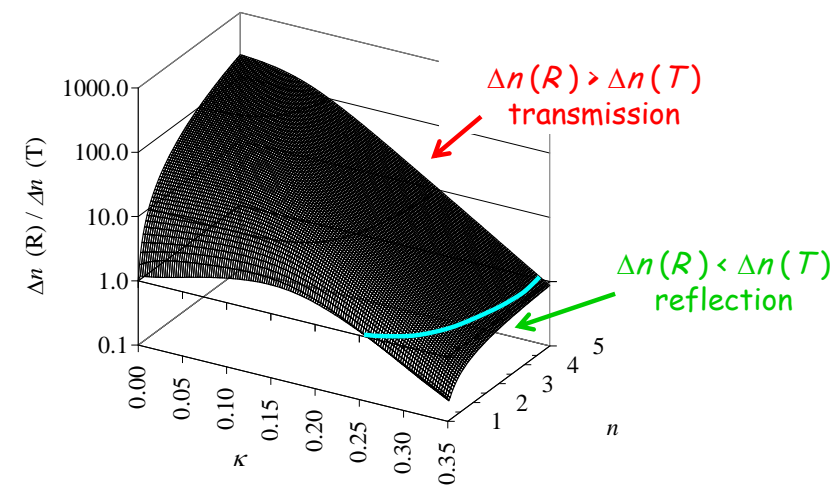

Fig. 4. Ratio $\Delta n(R) / \Delta n(T)$ versus $n$ and $\kappa$ for $\beta=20$.

Then we take the ratio of the $\Delta n$ 's calculated in reflection and transmission. If this ratio is larger than 1 , transmission gives better precision and should be selected as a method of interest. Figure 4 presents the ratio $2 \mathrm{D}$ map for $\beta=20$ corresponding, for example, to a $1-\mathrm{mm}$ thick sample studied at $1 \mathrm{THz}$. For transparent or moderately transparent materials (i.e., in the present case, for $\kappa<0.25$, which corresponds to $\alpha<100 \mathrm{~cm}^{-1}$ at $1 \mathrm{THz}$ ) transmission is as expected better, while reflection is preferable for highly absorbing materials. The effect of the refractive index is less pronounced than absorption.

Finally, we summarize our results by plotting on a $2 \mathrm{D}$ graph (see Fig. 5) the curve $\Delta n(T)=\Delta n(R)$ versus $n$ and $\kappa$. We treat different cases corresponding to $\beta$ varying from 1 to 20 , i.e. $d$ varying from $50 \mu \mathrm{m}$ to $5 \mathrm{~cm}$ at a $1-\mathrm{THz}$ working frequency. In the region "over" each curve, as regards to $\kappa, \Delta n(T)>\Delta n(R)$ and thus reflection is preferable to transmission. Let us notice that, in Fig. 5, we care about most of practical cases. Indeed, the sample thickness varies from a few tens of $\mu \mathrm{m}$ to several $\mathrm{cm}$, and $n$ from 1 to 3.5 . At $1 \mathrm{THz}$, the $\kappa$ range (0.001-10) corresponds to an absorption coefficient $\alpha$ ranging approximately from 0.4 to $4000 \mathrm{~cm}^{-1}$.

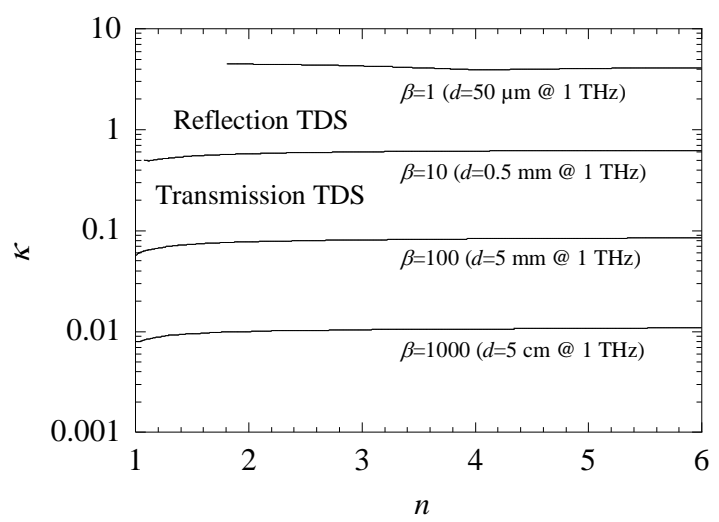

Fig. 5. Curves $\Delta n(R)=\Delta n(T)$ versus $n$ and $\kappa$ for different values of $\beta$. For a given $\beta$, reflection TDS is more suited over the curve, while transmission TDS has to be used under the curve, as indicated for $\beta=10$.

Here again, the choice between reflection and transmission is not strongly governed by the value of the refractive index. On the other hand, the thicker the sample, the smaller should be $\kappa$ to make the transmitted signal strong enough for transmission TDS to be more precise than reflection TDS.

In conclusion, we have studied the noise-limited precision in the extraction of the complex refractive index from THz-TDS measurements performed either in transmission or reflection. The results of our modelling study are experimentally validated. Finally, we give rules for selecting the technique most adapted to the samples to be characterized.

\section{References}

[1] M. van Exter, D. Grischkowsky, Appl. Phys. Lett. 56, 1694 (1990); Phys. Rev. B 41, 12140 (1990).

[2] Sensing with Terahertz Radiation, ed. D. Mittleman, Springer Series in Optical Sciences Vol. 85, Berlin (2003).

[3] For example: www.menlosystems.com; www.rainbowphotonics.com; www.teraview.com; www.ekspla.com; www.picometrix.com., www.zomega-terahertz.com.

[4] L. Duvillaret, F. Garet, J.-L. Coutaz, IEEE J. Sel. Topics in Quant. Electron. 2, 739 (1996).

[5] T. Dorney, R. Baraniuk, D. Mittleman, J. Opt. Soc. Am. A 18, 1562 (2001); I. Pupeza, R. Wilk, M. Koch, Opt. Exp. 15( 7), 4335 (2007).

[6] L. Thrane, R.H. Jacobsen, P.U. Jepsen, S.R. Keiding, Chem. Phys. Lett. 240, 330 (1995); S.C. Howells, L.A. Schlie, Appl. Phys. Lett. 69, 550 (1996).

[7] P. Uhd Jepsen, B.M. Fischer, Opt. Lett. 30, 29 (2005).

[8] L. Duvillaret, F. Garet, J.-L. Coutaz, J. Opt. Soc. Amer. B17, 452 (2000). 